\title{
Action Recognition and Understanding using Motor Primitives
}

\author{
Ville Kyrki \\ Department of Information Technology \\ Lappeenranta University of Technology, Finland \\ kyrki@lut.fi
}

\author{
Isabel Serrano Vicente, Danica Kragic, Jan-Olof Eklundh \\ Computational Vision and Active Perception Lab \\ Centre for Autonomous Systems, KTH, Stockholm, Sweden \\ isasevi,danik, joe@nada.kth.se
}

\begin{abstract}
We investigate modeling and recognition of arm manipulation actions of different levels of complexity. To model the process, we are using a combination of discriminative support vector machines and generative hidden Markov models. The experimental evaluation, performed with 10 people, investigates both definition and structure of primitive motions as well as the validity of the modeling approach taken.
\end{abstract}

\section{INTRODUCTION}

Recognition of human activity has been used extensively for robot task learning through imitation and demonstration, [1]-[8]. The discovery of mirror neurons in monkey's brain has introduced new hypotheses and ideas about the process of imitation and its role in the evolution [9], [10]. It has been shown in [11] that an action perceived by a human can be represented as a sequence of action units. This motivates the idea that the action recognition process may be considered as an interpretation of the continuous human behavior which, in its turn, consists of a sequence of action primitives [5]. In this work, we are investigating non-cyclic actions, with a focus on manipulation actions, which have not been studied extensively earlier. The specific questions that the study aims to answer are:

1) Can individual semantic actions be considered as manipulation primitives?

2) If not, can these be broken down into primitives? and

3) How can new actions emerge from known primitives?

For this purpose, we consider five different manipulation actions performed on an object: a) pick up, b) rotate, c) push forward, d) push to side, and e) move to side by picking up. To increase the variability, each action is performed by 10 different people in 12 different conditions. We strongly believe that the findings of the study will facilitate imitation learning in robots, both in terms of what vocabulary of primitives to learn and how to combine the individual primitives in order to form more complex actions. To model the process, we are using a combination of discriminative and generative models. A support vector machine (SVM) is used to model and recognize individual primitives, while the sequences of primitives are modeled using a hidden Markov model (HMM).

This paper is organized as follows. First, we review related work in Sec. II. Then, the theoretical basis for the work and two different approaches for primitive based modeling of manipulation actions are described in Sec. III. Section IV describes our experimental system. Experiments and their results are reported in Sec. V. Finally, the results are discussed and a conclusion given in Sec. VI.

\section{RELATED WORK}

In [4], a framework for acquiring hand-action models by integrating multiple observations based on gesture spotting is proposed. [5] approaches the task learning problem by proposing a system for deriving behavior vocabularies or simple action models that can be used for more complex task extraction and learning. [8] presents a learning system for one and two-hand motions where the robot's body constraints are considered as a part of the optimal trajectory generation process. An interesting trend to note here is that most of the studies are based on a single user generated motion. A natural question to pose here is how the underlying modeling methods scale and apply for cases when the robot is supposed to learn from multiple teachers. The experimental evaluation conducted in our work is based on 10 people.

Related to the theoretical framework used in this work, support vector machine (SVM) has been applied to several different application areas. Two very common data types are visual and speech data [12], [13]. Earlier work with SVMs [14] presented one drawback when working with sequential data, namely that SVM lacks a way of handling the time dependencies in the data. In order to use time sequences as SVM input, variable length time sequences can be either normalized to same length before applying the SVM. Another approach is to embed dynamic time warping (DTW) directly into the SVM kernel function [15]. Third, probably most common way to handle the "time problem" The most common approach is to combine a SVM with Hidden Markov Models (HMM) [13], [14], [16]. SVM is still used to classify single points or brief time windows, but the output of the SVM is then used an input to a HMM which then finds the most probably path or sequence in consideration of time. In action recognition and understanding, it is most common to take a holistic approach, that is, to consider all measurements as a single feature. This in contrast to speech recognition where it is common to divide the data into individual phonetics or words. From the point of view of imitation learning or "learning by showing", the primitives are an attractive option since they can alleviate mapping motion from humans to robots which differ in their embodiment. In addition, having a common vocabulary of primitives can aid in task understanding and planning as the task can be then described as a sequence of events. For this reason, we now concentrate on this body of work. Ogawara et al. [17] propose to extract primitive actions by learning several HMMs and then cluster these HMMs such that each cluster represents one primitive. Thus, variability within each primitive can be modeled as each cluster can contain 
several examples. Vecchio et al. [18] model two-dimensional drawing actions as dynamical systems and classify and segment motions according to a priori known motion classes. Segmentation of repetitive movements and stochastic parsing have been studied in [19]-[21].

\section{MODELING METHODS}

We present the theoretical basis on recognizing individual primitives using SVMs and the time sequence modeling using hidden Markov models. Two approaches of primitive based modeling of actions are also described.

\section{A. Support vector machines}

The aim of support vector classification is to separate two classes, mapped into a high dimensional feature space, by a hyperplane with a maximal margin to both classes. The hyperplane is the decision boundary of the classifier with feature vectors on one side belonging to a first class and vectors on the other side to a second one. To represent complex decision boundaries, the mapping (kernel) from the original feature space to the high dimensional space is nonlinear. In this work, a standard SVM with Gaussian kernel is used.

To apply SVM classification for more than two classes, we take the one-against-one approach. That is, by denoting the number of classes by $k, k(k-1) / 2$ classifiers are trained using all pairs of classes. To classify a sample from an unknown class, it is classified by all classifiers, and each result is a vote for the class. Majority voting is used to decide the class of the sample. The one-against-one approach has been found very successful with SVMs but it suffers from increased number of individual classifiers when the number of classes is very high.

\section{B. Markov chain and hidden Markov models}

In this work, we are interested in time-homogeneous Markov chain models, that is, the state transition probabilities are invariant over time. Denoting the state $i$ by $\omega_{i}$, the time evolution of states can then be described using the state transition probabilities $P\left(\omega_{j}(t+1) \mid \omega_{i}(t)\right)=a_{i j}$. The states themselves are hidden, not directly observable. Instead, in each state, an observation $\mathbf{x}(t)$ is made. The observation depends only on the current state according to a selected probabilistic model, that is, $P\left(\mathbf{x}(t) \mid \omega_{i}(t)\right)=P\left(\mathbf{x} \mid \omega_{i}\right)$. If the set of observations $X$ is discrete and finite, $X=\left\{\mathbf{x}_{1}, \ldots, \mathbf{x}_{M}\right\}$, the observation probabilities can be written more shortly as $P\left(\mathbf{x}_{j} \mid \omega_{i}\right)=b_{i j}$. Finally, the probability of starting in state $\omega_{i}$ can be defined as $\pi_{i}=P\left(\omega_{i}(1)\right)$. Thus, the parameters can be collected to matrices $\mathbf{A}$ and $\mathbf{B}$ and a vector $\pi$.

Our objective is to model actions based on motor primitives that correspond to individual states of the HMM. A typical approach for using HMMs in recognition is to build a single HMM for each class to be recognized and then determine the class of an unknown sample by using the maximum likelihood method. In this work, we take another approach and represent the whole set of actions with a single HMM, such that different paths through the HMM correspond to different actions. This is because many actions contain similar parts. As an example see Fig. 1 where both rotating and pushing an object both require first the hand to approach the object. Our hypothesis is also that more complex actions can be modeled using a set of motor primitives. Thus, instead of making a choice between several HMMs, the most probable path through the HMM is sought. The path is found by the Viterbi algorithm [22], a dynamic programming based algorithm for determining the maximum likelihood path through a HMM given a sequence of observations $(\mathbf{x}(1), \mathbf{x}(2), \ldots)$. It finds the state sequence $(\omega(1), \ldots)$ for which $P(\mathbf{x}(1), \ldots, \mathbf{x}(T) \mid \omega(1), \ldots, \omega(T))$ is maximal.

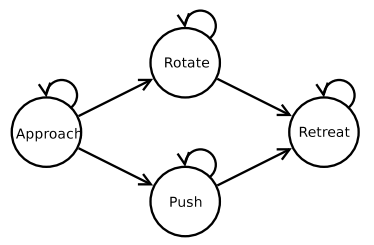

Fig. 1. Modeling two actions (rotate, push) using primitives.

To learn the HMM parameters initially, we take an alternative approach to the traditional Baum-Welch learning. We use labeled examples as training data, that is, for each time step, the current motor primitive is known. Then, the transition probability matrix A can be directly estimated from the training data, as if in the case of a Markov chain model instead of a HMM. We use the maximum likelihood estimate, in other words, the transition probabilities are calculated directly from the training data. The output of the SVM is used as the observations of the HMM. The observation probabilities need also be estimated as it is not expected that the classifier will be able to classify all samples correctly. Maximum likelihood estimation using the known correct classes is also used to estimate the observation probabilities. Therefore, the observation matrix $\mathbf{B}$ corresponds to the confusion matrix of the classifier.

\section{Action modeling}

The hypothesis in the modeling is that each of the manipulation primitives is generic and that their number is limited. However, the best applicable set of primitives is not known and one of the goals of this study is to inspect, how the manipulation actions can be considered in terms of primitives.

We investigate two different models of action representation, see Fig. 2. Approach 1 considers each of the manipulation actions as a primitive. In addition to the manipulation actions, two assisting actions, approach and remove are inherent in all action sequences (see Fig. 2). The assisting actions alleviate the segmentation of the manipulation part of the action. Approach 2 considers that the manipulation part of the action can be composed of multiple primitives. The model on the right in Fig. 2 can be chosen based on the knowledge that the rotation and moving the object require grasping. Our working hypothesis is that Approach 2 would be more effective in recognizing actions compared to the first approach. In addition it would allow learning of new actions based on the known primitives.

In both approaches, each action is represented by a separate path through the left-to-right Markov model. Considering Approach 2, to learn a new composite action, it is enough to learn the new sequence of primitives, if the primitives are 

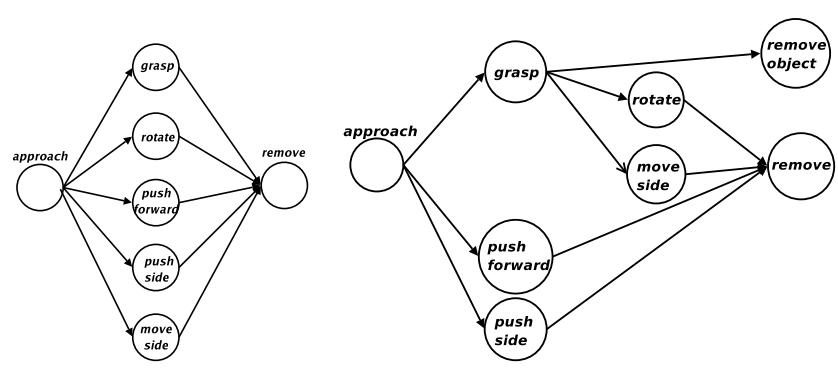

Fig. 2. (left): Actions as primitives; (right): Composite actions.

already known. If a hypothesis of the sequence of primitives is available, the only parameters that have to be learned are the transition probabilities of the model. However, having an unknown sequence, the only available information is the sequence of observations (SVM output) which contains uncertainty. As the transition probabilities are inherent to the underlying hidden states, not the symbols that are observed, the learning must be performed by considering the BaumWelch re-estimation (forward-backward algorithm) [22]. It should be noted that by initializing the estimation with nonzero probabilities only along the desired path, the estimation process will find the locally optimal probabilities within the path such that no new states will be introduced. If the observation probabilities of the primitives are also known in advance, only the transition matrix of the HMM needs to be updated in the estimation.

Upper part of Fig. 3 shows the composite action model without the move to side primitive. The lower part of the same figure demonstrates now a single possible representation of the move to side primitive. Note that now the new primitive is described fully by existing primitives. The transition probabilities for the new primitive can be estimated as discussed above. After learning a model for a new action,
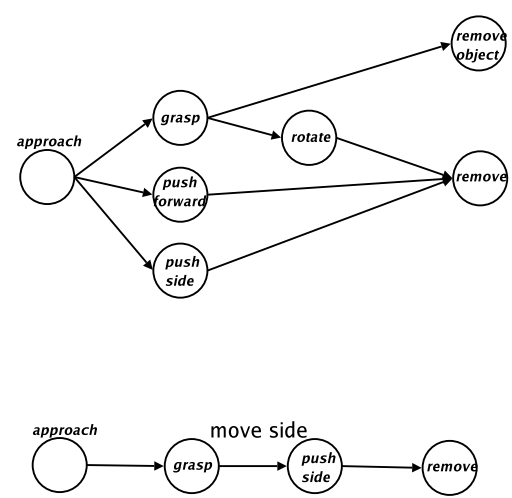

Fig. 3. Learning new composite actions.

the state transition probabilities of the model containing all actions must be updated according to that of the new action. During the process, new state transitions will be introduced in the model. This is illustrated in Fig. 4. The probabilities can be updated by weighted averaging of the transition probabilities from a state given the two models, with weights given by the number of actions using that state in that particular model. Thus, the upper model of Fig. 3 would have twice the weight compared to the lower one for paths leaving grasp because in the upper one there are two

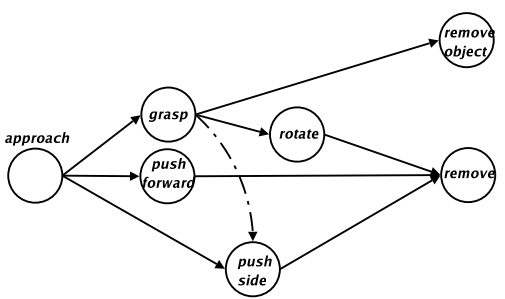

Fig. 4. Embedding a new action.

actions using the state. To determine the best sequence of primitives for a new action, exhaustive search can be used if the number of primitives is relatively low. Otherwise, search and pruning techniques would be necessary. However, the classification results of individual time instants give a strong cue as to which primitives are present in an unknown action.

\section{SYSTEM AND IMPLEMENTATION}

Five different actions are considered: a) pick up an object from a table, b) rotate an object on a table, c) push an object forward, d) push an object to the side, and e) move an object to the side by picking it up. To include variation in the actions, each action is performed in 12 different conditions, namely on two different heights, two different locations on the table, and having the demonstrator stand in three different locations (0, 30, 60 degrees) (see Fig. 5). All actions are demonstrated by 10 different people.

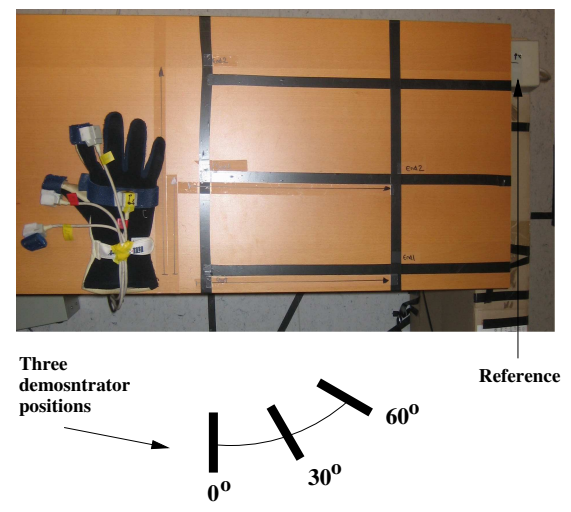

Fig. 5. Glove with the sensors, the table and the three demonstrator locations.

\section{A. Sensors and data}

The movement is measured using the Nest-of-Birds magnetic sensors. The test subject is endowed with four sensors each registering their full 3-dimensional pose with respect to a reference, see Fig. 5. The sensors are located on: a) chest, b) back of hand, c) thumb, and d) index finger. The chest sensor is used to provide a reference to the demonstrator position while the back of the hand can be used as a reference for the thumb and index finger. The measured sequences have been annotated by hand such that the current action primitive is known for training.

\section{B. System overview}

The goal of the system is to recognize actions, while this study also tries to reveal, how suitable primitive based 
techniques are for action description of manipulation actions. An overview of the system is given in Fig. 6. After preprocessing the data for noise removal, the primitives are recognized by an SVM and its output is then fed to an HMM which describes the time evolution. As the true action primitives are known, SVMs can be directly trained. A hidden Markov model is then used to describe the temporal sequence of primitives. The lower part of the system in Fig. 6 is concerned with the learning of new actions based on known primitives. In that case, the models are learned through the Baum-Welch re-estimation.

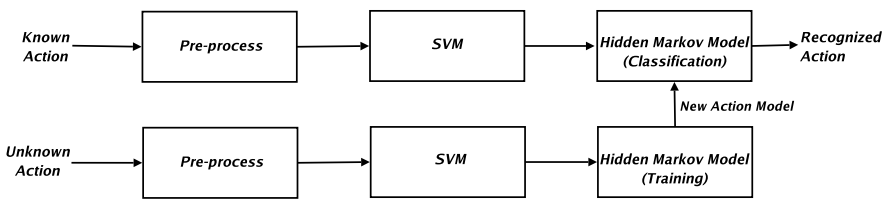

Fig. 6. System overview.

\section{Pre-processing}

The following sensor measurements are used:

- position of the hand relative to the chest: $x, y$ and $z$

- position of the index relative to the hand: $x, y$ and $z$

- position of the thumb relative to the hand: $x, y$ and $z$

- velocity of the hand: $v_{x}, v_{y}$ and $v_{z}$.

We start by applying the median filter of length 7 twice to the data so to eliminate the noise peaks. After filtering, the hand and finger locations were transformed into the chest reference frame. Next, the position of both the thumb and index was calculated with respect to the back of the hand. A Gaussian filter was then applied for the finger positions to reduce the noise, which was found to be most apparent in the finger position measurements. The velocity was estimated by time differences between two consecutive time instants. It was then filtered by a Gaussian filter to decrease the noise due to the differential nature of the estimation process. Finally, every dimension was linearly scaled to interval $[0,1]$.

The effect of the preprocessing before scaling is illustrated in Fig. 7, which demonstrates that while the spurious peaks are removed, the overall shape of the trajectory is not changed.

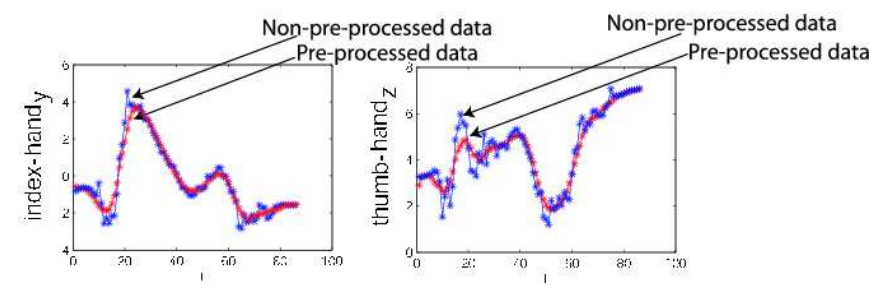

Fig. 7. Filtering for noise removal.

\section{EXPERIMENTAL EVALUATION}

We start by presenting the results of Approach 1, where each action is considered a separate primitive. Then, the actions are modeled as sequences of primitives, Approach 2. Finally, we study the capabilities of modeling a new action based on learned primitives. All actions were performed by 10 people in 12 different conditions, resulting in 120 different samples. The demonstrators were given only oral explanations of the task and for that reason, the inter-personal variance in the trajectories was high. This approach was taken to emphasize our goal of understanding actions instead of just tracking the movement. For SVM learning the training sequences were classified and segmented by a human. That result was also used as a ground truth for the experiments. In the following results, leave-one-out testing is always used where not indicated otherwise. Thus, one person was left out of the training set, that person was used to test the system, and this was repeated for all persons. Average performance is then reported.

\section{A. Actions as primitives}

In Approach 1, each manipulation action is a separate primitive. Here, the assisting primitives for approach and remove are present in all actions. The action model used can be seen in Fig. 2. The results of experiments are presented in Fig. 8. The upper table shows the confusion matrix for the SVM classification for each time instant. The rows correspond to the ground truth and the columns are the SVM output. It can be seen that some primitives (push forward, rotate, remove) are classified quite well for even considering only one time instant at a time. In contrast, two primitives, push side, move side seem to be overlapping in their representation as they are often confused with each other. This confusion is not surprising as the training data was overlapping for the two different primitives due to the high inter-personal variance of how the actions were performed. For that reason, it is possible that one person's move side was very similarly to another's push side.

Also the assisting primitives approach, remove were confused with each other. A more detailed analysis of the results revealed that this happened particularly when the movement was very slow. This explains the confusion, because with slow movements the velocity can not be estimated reliably enough in order to be used for discriminating between these two. Finally, the grasp primitive was confused quite often with rotate, move side. This is most likely the result that both of these two primitives also involve grasping. Thus, these primitives can not be recognized reliably considering single time instants.

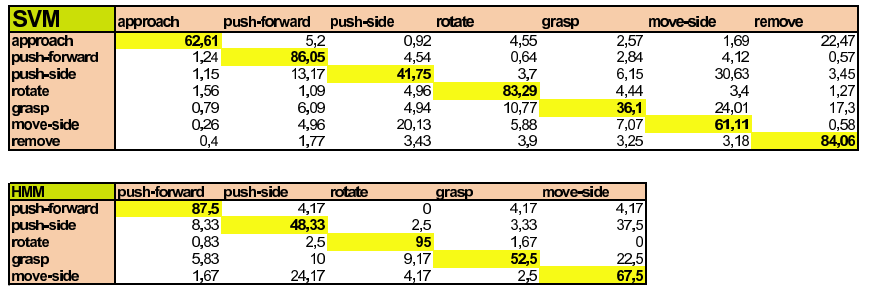

Fig. 8. Approach 1. Actions as primitives.

Next, the recognition results were used as an input to the HMM. The results of Viterbi based recognition of actions of the HMM are given in the lower table of Fig. 8. The ground truth is given again in the first column. Note that here each sequence is recognized as belonging to one of the actions instead of labeling all time instants. However, the Viterbi algorithm also gives the most probable primitive for each time instant such that the manipulation part can be segmented 
from the assisting primitives. The confusion matrix in Fig.8 again supports our earlier results that the pair push side-move side is difficult to recognize from each other. However, it can be argued that because also the semantic meanings of the two actions are similar, these errors could be tolerated, at least to some extent, in action understanding. Another finding is that grasp action could not be recognized individually as the same primitive also exists in other actions.

\section{B. Actions as composites}

It is evident from the previous experiment that considering the actions themselves as individual primitives did not yield good results. Next, the actions were modeled in a composite structure of primitives. Our approach was to model the individual primitives such that they had semantic meaning. The model is shown on right in Fig. 2. One new state, remove with object, was introduced by the argument that the end state of the environment is different in the case the person is holding the object in the end. This is the end state only for the grasp action. In addition, the structure of the model was changed such that all actions requiring grasping employ first the grasp primitive before the second manipulation primitive.

Fig. 9 presents the confusion matrix for SVM classification as well as the recognition result by the HMM. The SVM classification results change significantly for two primitives, grasp, remove. The results of recognizing grasp increase significantly, as it is no longer confused with other actions requiring grasping. Based on this result, we can hypothesize that motion primitives exist and that grasp can be considered as one. For the remove primitive the recognition rate decreases, because a very similar new primitive remove with object was introduced. It should be noted that SVM still confuses push side with move side.

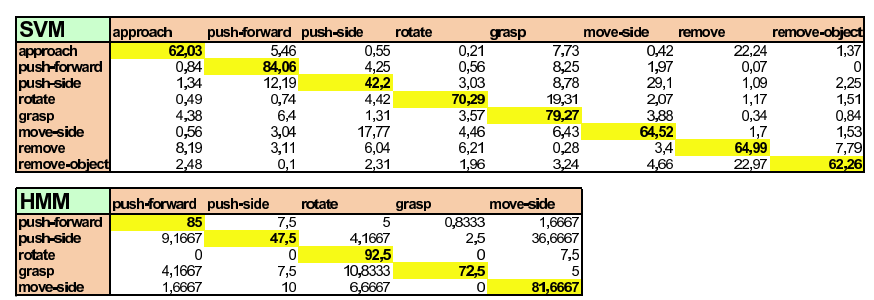

Fig. 9. Approach 2: Composite actions.

The confusion matrix for the HMM (Fig. 9) has improved significantly for two actions, grasp, move side, compared to Approach 1. For move side, this result can be explained by the fact that grasp primitive is required for all actions in this class, making it easier to discriminate between push side and move side. An important note is that the SVM classification result did not improve from Approach 1, but this results from enforcing a particular time sequence of events for the action. It should be, nevertheless, noted that push side, move side are still confused, for the reason given in Sec. V-A. For grasp primitive, the improvement is due to improvement in the SVM classification discussed above.

\section{Modeling a new action}

We now investigate if new actions can be modeled using learned primitives. From the earlier results it is known that the move side action is similar to push side. We performed the investigation by removing the move side actions from the training data of the SVM. Thus, the SVM only learned the other primitives. Our goal was then to see which sequential model using the other primitives would be optimal for modeling the move side actions. The experiment was begun by modeling the system (without move side) in the way shown in upper part of Fig. 3. Thus, the SVM was also trained without any of the move side data. The performance results for this model are shown in Fig. 10. The classification performance improves for those primitives, which were earlier confused with the move side primitive.

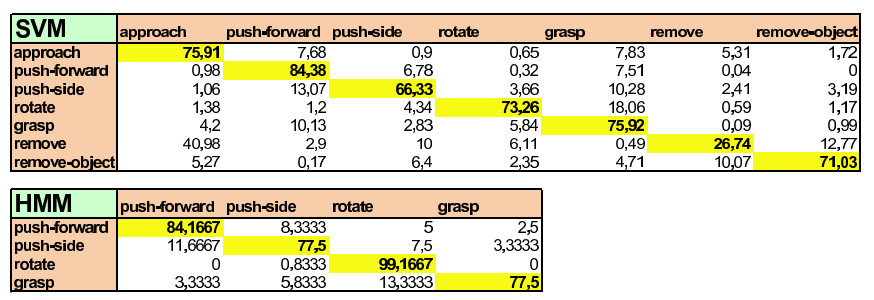

Fig. 10. Modeling a new action: Before new action.

The best left-to-right state model for move side was found among all 3 and 4 state models. The starting state was fixed to approach and the end state to remove in order to constrain the problem to determining the manipulation primitives used. Exhaustive search was used by enumerating all possible models. Each model was trained using the BaumWelch re-estimation as described in Sec. III-C using all of the move side sequences as input. Note that now the sequence was not segmented by hand into primitives but the underlying states were considered hidden, and the SVM confusion matrix in Fig. 10 was used as the model for the measurement uncertainty of the HMM. The goodness of fit for each model was evaluated by calculating the joint probability of observing all the training sequences given the new model, where the forward-algorithm [22] was used for each individual sequence. These results are given in Fig. 11 where the upper part show the log-probabilities for each of the 12 different 3 and 4 state models. The model that fits the data best is approach - grasp - push side - remove, shown in the bottom of Fig. 3. This model seems to grasp the semantic meaning of the action very well. If the new model is embedded into the existing HMM, as described in Sec. III-C, the lower part of Fig. 11 presents the classification results of this HMM. The recognition rate of $62.5 \%$ is good considering that no data of the action sequences was used in the SVM training.

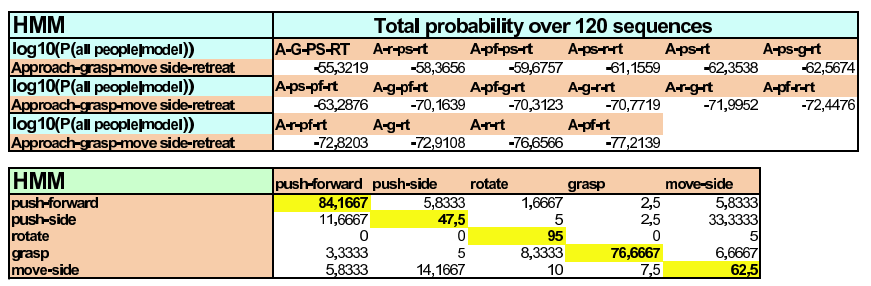

Fig. 11. Modeling new action: Best action, Classification in combined HMM.

To further examine the inter-personal variance in motion primitives, we repeated the experiment such that now all 
persons, including the test person, were used in the training of the SVM. Thus, it was supposed that if the hypothesis of actions consisting of primitives is valid, the recognition rate of individual primitives would increase also for the unknown actions where known primitives are used in unknown contexts. The results of this experiment are shown in Fig. 12. The recognition rate for the move side action increased from $62.5 \%$ to $77.5 \%$. This result can be considered remarkable because it suggests that to learn good models for complex actions for a wide variety of people, it is important to learn the individual ways of each person executing a certain primitive and that the sequences of primitives for particular semantic actions can be learned in general from data from other people demonstrating the same action.

\begin{tabular}{|c|c|c|c|c|c|}
\hline SVM+HMM & push-forward & push-side & rotate & grasp & move side \\
\hline push-forward & 95,83334 & 0,83333 & 3,33333 & 0 & 0 \\
\hline push-side & 4,16666 & 59,16667 & 0 & 0,83333 & 35,83334 \\
\hline rotate & 0 & 0 & 99,16667 & 0 & 0,83333 \\
\hline grasp & 0,83333 & 0 & 2,5 & 94,16667 & 2,5 \\
\hline move side & 0 & 6,66666 & 10,83334 & 5 & 77,5 \\
\hline
\end{tabular}

Fig. 12. Modeling new action: Classification with personal learning of primitives.

\section{DISCUSSION}

We have studied the recognition and understanding of manipulation actions performed by humans. While the literature in action recognition is large, there are not many extensive studies on the modeling of the manipulation actions, which have the characteristic of being typically very similar to each other. Similar to some other studies, we have considered a framework where the actions are composed of primitives. However, in contrast to others, we consider two alternative hypotheses: 1) individual actions can be considered manipulation primitives, and 2) manipulation actions should be broken down into primitives.

Based on initial results, we have realized that even quite simple manipulation actions consist of several primitives, which, however, might be common with other actions. The idea of composite actions is thus result of initial evaluation of the model "actions as primitives". We have also considered assisting primitives, such as approaching the object, which might not serve directly in the recognition of the action but which still can be useful in segmenting the manipulation.

Rather than using generative models for the whole action, SVM based discriminative models have been used for the recognition of individual primitives. This is because our focus is on action recognition and understanding rather than action synthesis. It should be noted that although in this paper the classification is done each time instant, the considerations apply to the case when short time windows are used instead of instants. Also, the ideas presented are by no means limited to a particular classifier (such as SVM) for the primitives.

The data for experiments was collected from 10 different demonstrators, each demonstrating the actions in several different conditions, and with only an oral explanation of the action given. Thus, the data had significant intra- and interpersonal variation. The most important findings of the experiments are that a) sequences of simple semantic primitives can be used in describing actions, b) inter-personal variations in primitives are significant, and c) actions learned as sequences of primitives from other demonstrators can be combined with knowledge of personal primitives to recognize new actions. Future work will study what new actions can be modeled with our current primitives, and what set of primitives would be appropriate to model a large variety of manipulation tasks typically performed by humans.

\section{REFERENCES}

[1] Y. Kuniyoshi, M. Inaba, and H. Inoue, "Learning by watching," in IEEE Transactions on Robotics and Automation, vol. 10(6), pp. 799$822,1994$.

[2] S. Schaal, "Is imitation learning the route to humanoid robots?," Trends in Cognitive Sciences, vol. 3, no. 6, pp. 233-242, 1999.

[3] A. Billard, "Imitation: A review," Handbook of brain thory and neural network, M. Arbib (ed.), pp. 566-569, 2002.

[4] K. Ogawara, S. Iba, H. Kimura, and K. Ikeuchi, "Recognition of human task by attention point analysis," in IEEE Int. Conf. on Intelligent Robot and Systems IROS'OO, pp. 2121-2126, 2000.

[5] O. C. Jenkins and M. J. Mataric, "Performance-derived behavior vocabularies: Data-driven acqusition of skills from motion," Int. Journal of Humanoid Robotics, vol. 1, pp. 237-288, Jun 2004.

[6] M. C. Lopes and J. santos Victor, "Visual transformations in gesture imitation: What you see is what you do," in IEEE Int. Conf. on Robotics and Automation, ICRA04, pp. 2375- 2381, 2003.

[7] S. Ekvall and D. Kragic, "Grasp recognition for programming by demonstration tasks," in IEEE Int. Conf. on Robotics and Automation, ICRA'05, pp. $748-753,2005$.

[8] S. Calinon, A. Billard, and F. Guenter, "Discriminative and adaptative imitation in uni-manual and bi-manual tasks," in Robotics and Autonomous Systems, vol. 54, 2005.

[9] V. Ramachandran, "Mirror neurons and imitation learning as the driving force behind the gerat leap forward in human evolution," Edge, vol. 69, 2000.

[10] L. Fadiga, L. Fogassi, V. Gallese, and G. Rizzolatti, "Visuomotor neurons: Ambiguity of the discharge or 'motor perception'?," Int. Journal of Psychophysiology, vol. 35, no. 2-3, pp. 165-177, 2000.

[11] D. Newton et al, "The objective basis of behavior unit," Journal of Personality and Social Pshychology, vol. 35, no. 12, pp. 847-862, 1977.

[12] P. Clarkson and P. J. Moreno, "On the use of support vector machines for phonetic classification," Compaq Computer Corporation, Cambridge Research Laboratory USA, 1999.

[13] M. Bartlett, G. Littlewort, B. Braathen, T. Sejnowski, and J. Movellan, "A prototype for automatic recognition of spontaneous facial actions," in Advances in Neural Information Processing Systems, NIPS 2003, pp. 1271-1278, 2002.

[14] S. E. Golowich and D. X. Sun, "A support vector/hidden Markov model approach to phoneme recognition," in ASA Proceedings of the Statistical Computing Section, pp. 125-130, 1998.

[15] H. Shimodaira, K. ichi Noma, M. Nakai, and S. Sagayama, "Dynamic time-alignment kernel in support vector machine," in Advances in Neural Information Processing Systems 14, NIPS2001, pp. 921-928, 2001.

[16] D. Surendran and G.-A. Levow, "Dialog act tagging with support vector machines and hidden Markov models," in Interspeech 2006 - ICSLP, (Pittsburgh, PA, USA), 2006.

[17] K. Ogawara, J. Takamatsu, H. Kimura, and K. Ikeuchi, "Modeling manipulation interactions by hidden Markov models," in IEEE/RSJ Int. Con. on Intelligent Robots and Systems, pp. 1096-1101, 2002.

[18] D. Del Vecchio, R. M. Murray, and P. Perona, "Decomposition of human motion into dynamics-bbased primitives with application to drawing tasks," Automatica, vol. 39, no. 12, pp. 2085-2098, 2003.

[19] C. Lu and N. Ferrier, "Repetitive motion analysis: Segmentation and event classification," IEEE Transactions on Pattern Analysis and Machine Intelligence, vol. 26, no. 2, pp. 258-263, 2004.

[20] Y. Ivanov and A. Bobick, "Recognition of visual activities and interactions by stochastic parsing," IEEE Transactions on Pattern Analysis and Machine Intelligence, vol. 22, no. 8, pp. 852-872, 2000.

[21] M. Yamamoto, H. Mitomi, F. Fujiwara, and T. Sato, "Bayesian classification of task-oriented actions based on stochastic contxt-free grammar," in Int. Conf. on Automatic Face and Gesture Recognition, (Southampton, UK), April 10-12 2006.

[22] L. R. Rabiner, "A tutorial on hidden Markov models and selected applications in speech recognition," Proceedings of the IEEE, vol. 77, no. 2, pp. 257-286, 1989. 